\title{
Serum Thioredoxin as a Diagnostic Marker for Hepatocellular Carcinoma in Cirrhotic Hepatitis C Patients
}

\author{
Tarek Sheta ${ }^{1, *}$, Mahmoud Selim², Mohammed Sabry ${ }^{3}$, Ahmed Saed $^{1}$ \\ ${ }^{1}$ Internal Medicine Department, Faculty of Medicine, Mansoura University, Mansoura, Egypt; \\ ${ }^{2}$ Internal Medicine Department, Faculty of Medicine, Delta University for Science \& Technology, Mansoura, Egypt; \\ ${ }^{3}$ Clinical Pathology, Specialized Medical Hospital, Mansoura University, Mansoura, Egypt.
}

\begin{abstract}
Background: Hepatocellular carcinoma (HCC) is the most frequent primary liver malignancy. Early detection of HCC is extremely important in improving the survival of patients. Alpha-fetoprotein (AFP) was commonly used as a predictor for HCC, but it was associated with low sensitivity and specificity. Thioredoxin (TRX) is a ubiquitous protein that was suggested to be elevated in cases with HCC.

Objective: To evaluate the value of serum thioredoxin as a diagnostic marker of HCC versus alpha-fetoprotein in cirrhotic HCV patients.

Materials and methods: This study included 96 patients divided into; groups I included, patients with liver cirrhosis, and group II included, patients with HCC on top of a cirrhotic liver. Both groups were successfully undergoing treatment of $\mathrm{HCV}$ with direct-acting antiviral (DAAs). Basic data, clinical examination, and laboratory analysis were obtained from all the cases. Human thioredoxin detection was done using Human TRX kits. Results: There is statistically significant increased TRX, AFP, APRI, and FIB4 among hepatocellular carcinoma group versus cirrhotic group. The ROC curve analysis demonstrated that TRX at a cut-off value of 198.19 (IU/ml) has $85.4 \%$ sensitivity and $89.6 \%$ specificity for differentiating HCC cases from cirrhotic cases with $89.1 \% \mathrm{PPV}, 86 \% \mathrm{NPV}$, and AUC equal to 0.841 . Both, AFP at a cut-off of $24(\mathrm{ng} / \mathrm{ml})$ and combined AFP and TRX had $93.8 \%$ sensitivity and $97.9 \%$ specificity with AUC equal to 0.99 for differentiating $\mathrm{HCC}$ cases from cirrhotic cases.

Conclusion: Thioredoxin is a novel biomarker that revealed good sensitivity in the prediction of HCC on top of liver cirrhosis especially if combined with AFP.
\end{abstract}

\section{Introduction}

$\mathrm{HCC}$ is the most frequent primary liver malignancy and one of the most common malignancies worldwide. HCC is considered as the sixth most common cancer type and as the

Keywords: Thioredoxin, hepatocellular carcinoma, cirrhosis, direct acting antiviral, alpha-fetoprotein.

Received: 27-1-2021 ; Accepted: 15-2-2021

* Corresponding author. Email: Drtarek_sheta@yahoo.com third cause of cancer-related death in the developed countries; more than a million people are dying yearly due to HCC in the Western countries ${ }^{1}$. In Egypt, it is believed now that, HCC is one of the common malignancies and a leading cause of death due to the high prevalence of cirrhosis related to chronic $\mathrm{HCV}^{2}$.

At diagnosis, the tumor has very often reached an advanced stage and curative treatment options are missing. Thus, early diagnosis would help the patient and prevent increasing health care costs ${ }^{3}$. The progression of liver disease into liver cancer is primarily monitored by serum levels of the oncofetal glycoprotein, alpha-fetoprotein, or the core fucosylated glycoform of AFP (AFP L3). AFP was considered a serum marker for screening patients at high risk for HCC, as well as for the diagnosis and monitoring of responses to HCC treatment for over 40 years ${ }^{4}$.

A significant increase in serum AFP level is detected in a considerable number of chronic liver diseases ${ }^{5-7}$. Therefore, the use of AFP as a primary screening test for $\mathrm{HCC}$ has been questioned, and raising the need for more sensitive and specific serum biomarkers for HCC are wanted. Thioredoxin is a ubiquitous protein with two redoxactive cysteine residues in the active center, having the amino acid sequence (-Cys-Gly-Pro-Cys-) ${ }^{8}$. The increased thioredoxin expression has been seen in a variety of malignancies, including HCC, and evidence suggests that it may be associated with aggressive tumor growth and poor survival ${ }^{9,10}$. This study was conducted to evaluate the value of serum thioredoxin as a diagnostic marker of $\mathrm{HCC}$ versus alpha-fetoprotein in cirrhotic HCV patients.

\section{Materials and methods \\ Study settings and duration}

This study was conducted in the Internal Medicine department, Specialized medical hospital, Mansoura University during the period from December 2018 to December 2019.

\section{Patients}

This study included 96 patients with chronic HCV infection, the patients were classified into two groups; group I included 48 patients with liver cirrhosis who successfully underwent treatment of $\mathrm{HCV}$ with directacting antiviral and group II included 48 patients with HCC on top of the cirrhotic liver who were successfully treated of HCV with DAAs. 


\section{Original Article}

We included patients with the following criteria; Age > 18 years, successfully treated of $\mathrm{HCV}$ with DAAs (confirmed by negative quantitative PCR for HCV RNA after at least 6 months from the end of DAAs therapy).

Liver cirrhosis diagnosed by clinical, biochemical, and abdominal ultrasonographic findings, and HCC patients are diagnosed by abdominal ultrasound and Triphasic Computerized Tomography.

Diagnosis of HCC was based on the characteristic arterial enhancement and early washout in the delayed phase ${ }^{11}$. The patients with the following conditions were excluded; prior liver transplant, prior treatment of HCC (either surgical, interventional, or medical), malignancies other than HCC, presence of severe co-morbidity, noncompliant patients, and HBV hepatitis patients.

\section{Methodology}

All cases were subjected to complete history taking and abdominal examination. Full laboratory investigations were done in all the cases including; $\mathrm{CBC}$, polymerase chain reaction (PCR) to detect HCV RNA viral load, serum ALT, AST, serum bilirubin, serum albumin, prothrombin time and INR, serum creatinine, alpha-fetoprotein, hepatitis B surface antigen (HBsAg) and hepatitis B core immunoglobulin $\mathrm{G}$ (HBcIgG) and hepatitis $\mathrm{C}$ virus antibody by ELISA technique.

Fibrosis-4-score (FIB4) was calculated for the cases and mild fibrosis was diagnosed at value $<1.45$ and score $>3.25$ was diagnostic for advanced fibrosis ${ }^{12}$. Abdominal and pelvic ultrasound was performed for all the cases and triphasic abdominal CT after oral water and IV contrast was performed for detection of cases of HCC. All patients were classified according to the Child-Pugh Score.

Human thioredoxin detection was done using Human TRX kits (Bioassay technology laboratory Shanghai Crystal Day Biotech Co., LTD) Cat. No: E1452Hu. To perform the assessment, $3 \mathrm{ml}$ of venous blood collected from all patients by clean venipuncture using plastic disposable syringes.

Each blood sample was delivered into a pyrogen/endotoxin-free plastic collecting tube. Blood was allowed to clot before centrifugation for 10 minutes at approximately $3000 \mathrm{rpm}$ and serum was taken and stored frozen at $-70^{\circ} \mathrm{C}$ for assay of serum thioredoxin

\section{Statistical analysis}

Data entered and analyzed using Microsoft Excel software. Data were then imported into Statistical Package for the Social Sciences (SPSS 21.0, IBM/SPSS Inc., Chicago, IL) software for analysis. Baseline characteristics of the study population were presented as frequencies and percentages $(\%)$ or mean values and standard deviations (SD) or median and range (after testing of normality by Kolmogorov-Smirnov and Shapiro-Wilk's tests). For comparison of data, Chi-Square test was used to compare two independent groups of qualitative data and Monte Carlo test as the correction for Chi-Square test when more than $25 \%$ of cells have to count less than 5 in tables. For quantitative data, independent-Samples t-test and MannWhitney $U$ test were used to compare two groups of

\section{Medical Journal of Viral Hepatitis (MJVH)}

parametric and non-parametric quantitative data respectively.

Spearman's rank-order correlation is used to determine the strength and direction of a linear relationship between two non-normally distributed continuous variables and/or ordinal variables (r). The diagnostic performance of a test or the accuracy of a test to discriminate diseased cases from non-diseased cases is evaluated using Receiver Operating Characteristic (ROC) curve analysis. Sensitivity and Specificity were detected from the curve and PPV, NPV and accuracy were calculated through cross-tabulation. Binary stepwise logistic regression analysis was used for the prediction of independent variables of a binary outcome. For all tests, $\mathrm{P}$ values $<0.05$ are considered significant.

\section{Results}

There is no statistically significant difference between studied groups as regards, age, sex, and smoking habits. The majority of patients with hepatocellular carcinoma were residing in rural areas. Moreover, there is no statistically significant difference between studied groups regarding body mass index, serum creatinine, albumin, total bilirubin, INR, hemoglobin, total leucocytic count, platelet count, and Child-Pugh score.

There was a significant increase in ALT and AST levels among hepatocellular carcinoma versus cirrhotic patients. Furthermore, there was a statistically significant increase in TRX, AFP, APRI, and FIB4 among the hepatocellular carcinoma group compared to the cirrhotic group (Tables 1). The ROC curve analysis (Tables 2) demonstrated that TRX at a cut-off value of 198.19 (IU/ml) has $85.4 \%$ sensitivity and $89.6 \%$ specificity for differentiating HCC cases from cirrhotic cases with $89.1 \% \mathrm{PPV}, 86 \% \mathrm{NPV}$, and AUC equal to 0.841 . The combined AFP and TRX had a sensitivity and specificity (93.8\% and $97.9 \%$ respectively) and AUC equal to 0.99. (Figure 1).

Using multivariate analysis for detection of hepatocellular carcinoma predictors, AFP and TRX are significant predictors of hepatocellular carcinoma with increased risk 1.16 and 1.01 , respectively for every increase of one unit in their values. The overall $\%$ predicted was 95.8\% (Table 3). TRX has a statistically significant positive correlation with AFP in the HCC group ( $p<0.001)$, however, in cirrhotic patients a nonsignificant correlation was found $(p=0.27$ ) (Table 4).

In patients with HCC, TRX had statistically significant positive correlation with Child Pugh classification ( $p=$ 0.34 ), focal lesion number and size ( $p=<0.001$ for each). AFP have also a statistically significant positive correlation with focal lesion number and size $(p=<.001$ and $<.0001$ respectively). In cirrhotic group, TRX had statistically significant positive correlation with INR $(p=0.007)$, Child Pugh classification $(p<0.001)$, and negative correlation with albumin $(p=-0.006)$. AFP have also a statistically significant positive correlation with ALT, AST, albumin and hemoglobin $(p=0.007, p=0.008, p=.031$ and $p=$ .041 respectively) (Tables 5). 
Table 1. Clinical and laboratory data of the studied groups.

\begin{tabular}{|c|c|c|c|}
\hline & Cirrhotic group $N=48$ & $\mathrm{HCC} \mathrm{N}=48$ & $P$-value \\
\hline Age (years) & $60.89 \pm 4.85$ & $62.06 \pm 3.72$ & 0.19 \\
\hline $\begin{array}{l}\operatorname{Sex}(\mathbf{N} / \%) \\
\text { Female } \\
\text { Male }\end{array}$ & $\begin{array}{l}18(37.5) \\
30(62.5)\end{array}$ & $\begin{array}{l}12(25.0) \\
36(75.0)\end{array}$ & 0.18 \\
\hline $\begin{array}{l}\text { Residence (N/\%) } \\
\text { Rural } \\
\text { Urban }\end{array}$ & $\begin{array}{l}20(41.7) \\
28(58.3)\end{array}$ & $\begin{array}{l}33(68.8) \\
15(31.2)\end{array}$ & 0.008 \\
\hline $\begin{array}{l}\text { Smoking }(\mathbf{N} / \%) \\
\text { Negative } \\
\text { Positive }\end{array}$ & $\begin{array}{l}39(81.2) \\
9(18.8)\end{array}$ & $\begin{array}{l}33(68.8) \\
15(31.2)\end{array}$ & 0.16 \\
\hline BMI $\left(\mathrm{kg} / \mathrm{m}^{2}\right)$ & $29.94 \pm 2.18$ & $29.76 \pm 2.12$ & 0.69 \\
\hline Serum creatinine & $1.12 \pm 0.16$ & $1.17 \pm 0.16$ & 0.11 \\
\hline $\operatorname{ALT}(\mathbf{U} / \mathbf{L})$ & $30.0(8.0-65.0)$ & $79.0(15.0-132.0)$ & $<0.001$ \\
\hline AST (U/L) & $48.0(30.0-94.0)$ & $108.0(41.0-188.0)$ & $<0.001$ \\
\hline $\operatorname{Albumin}(g / L)$ & $2.69 \pm 0.43$ & $2.70 \pm 0.37$ & 0.93 \\
\hline Total bilirubin (mg/dl) & $1.96(0.5-9.8)$ & $1.77(0.75-5.32)$ & 0.51 \\
\hline INR & $1.58 \pm 0.35$ & $1.56 \pm 0.28$ & 0.63 \\
\hline Hemoglobin (gm/dl) & $9.41 \pm 1.71$ & $9.84 \pm 1.73$ & 0.23 \\
\hline $\begin{array}{l}\text { Total leucocytic count } \\
\left(10^{3} / \mathrm{mm}^{3}\right)\end{array}$ & $6.32 \pm 1.61$ & $6.77 \pm 2.11$ & 0.25 \\
\hline Platelet count $\left(10^{3} / \mathrm{mm}^{3}\right)$ & $81.0(31.0-142.0)$ & $80.5(30.0-123.0)$ & 0.49 \\
\hline TRX (IU/L) & $157.92(20.85-13.75)$ & $338.06(105.65-99.8)$ & $<0.001$ \\
\hline $\operatorname{AFP}(\mathbf{n g} / \mathrm{ml})$ & $3.15(0.60-45.2)$ & $124.0(7.0-790.0)$ & $<0.001$ \\
\hline APRI & $1.5(0.7-6.6)$ & $3.7(0.9-8.6)$ & $<0.001$ \\
\hline FIB4 & $6.19(3.09-20.01)$ & $9.97(3.1-24.72)$ & $<0.001$ \\
\hline $\begin{array}{l}\text { Child-Pugh score }(\mathrm{N} / \%) \\
\text { A } \\
\text { B } \\
\text { C }\end{array}$ & $\begin{array}{c}15(31.2) \\
31(64.6) \\
2(4.2)\end{array}$ & $\begin{array}{c}12(25.0) \\
33(68.8) \\
3(6.2)\end{array}$ & 0.74 \\
\hline
\end{tabular}

ALT, alanine aminotransferase; AST, aspartate aminotransferase; INR, international normalized ratio

Table 2: Level and area under ROC of TRX, AFP, and combined TRX with AFP for differentiating HCC cases from cirrhotic cases.

\begin{tabular}{lccccccc}
\hline & $\begin{array}{c}\text { AUC } \\
(\mathbf{9 5 \%} \mathbf{C I})\end{array}$ & $\begin{array}{c}\text { Cut off } \\
\text { point }\end{array}$ & Sensitivity & Specificity & PPV & NPV & Accuracy \\
\hline TRX (IU/m) & $\begin{array}{c}0.841 \\
(0.754-0.927)\end{array}$ & 198.19 & $85.4 \%$ & $89.6 \%$ & $89.1 \%$ & $86.0 \%$ & $87.5 \%$ \\
\hline AFP $(\mathbf{n g} / \mathbf{m l})$ & $\begin{array}{c}0.99 \\
(0.987-1.0)\end{array}$ & 24.0 & $93.8 \%$ & $97.9 \%$ & $97.8 \%$ & $94.0 \%$ & $95.3 \%$ \\
$\begin{array}{l}\text { Combined } \\
\text { AFP and TRX }\end{array}$ & $\begin{array}{c}0.99 \\
(0.98-1.0)\end{array}$ & 0.5427 & $93.8 \%$ & $97.9 \%$ & $97.8 \%$ & $94.0 \%$ & $95.3 \%$ \\
\hline
\end{tabular}


Roc Curve

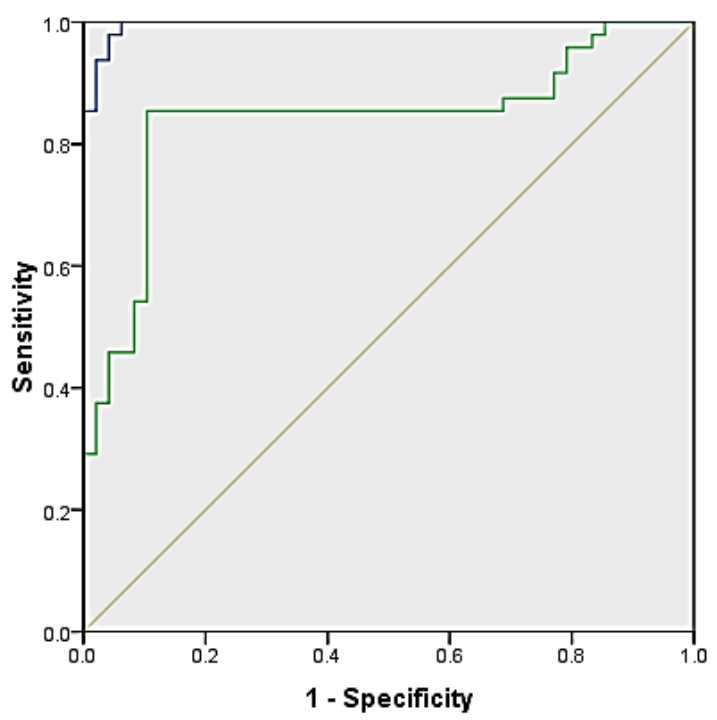

Source of the Curve - $\mathrm{AFP}$

Reference Lin

Figure 1. ROC curve of AFP \& TRX combined markers in differentiating HCC \&cirrhotic group.

Table 3. Multivariate analysis for detection of hepatocellular carcinoma predictors

\begin{tabular}{lccc}
\hline Predictors & B & $\boldsymbol{P}$ & $\begin{array}{c}\text { Odds ratio } \\
\text { (95\% CI) }\end{array}$ \\
\hline ALT (U/L) & 0.104 & 0.58 & $1.11(0.77-1.61)$ \\
AST (U/L) & 0.191 & 0.06 & $1.21(0.99-1.48)$ \\
\hline AFP (ng/l) & 0.143 & 0.002 & $1.16(1.06-1.26)$ \\
APRI & 0.66 & 0.07 & $1.94(0.958-3.94)$ \\
TRX (IU/ml) & 0.011 & $<0.001$ & $1.01(1.01-0.01)$ \\
FIB4 & 1.921 & 0.23 & $6.83(0.29-89.51)$ \\
Overall \% predicted $=\mathbf{9 5 . 8 \%}$ & & & \\
Constant $=\mathbf{- 5 . 2 9} \boldsymbol{p}<\mathbf{0 . 0 0 1 *}$ & & & \\
\hline
\end{tabular}

Table 4. Correlation between AFP \& TRX among hepatocellular carcinoma \& cirrhotic cases

TRX (IU/L)

\begin{tabular}{lccc}
\hline & & Cirrhotic group & HCC \\
\hline \multirow{2}{*}{ AFP } & $\mathrm{R}$ & -0.16 & 0.56 \\
& $P$ & 0.27 & $<0.001$ \\
\hline
\end{tabular}


Table 5. Correlation between studied markers and demographic \& laboratory data among hepatocellular carcinoma and cirrhosis cases.

\begin{tabular}{|c|c|c|c|c|c|c|c|c|}
\hline & \multicolumn{4}{|c|}{ HCC cases } & \multicolumn{4}{|c|}{ Cirrhotic cases } \\
\hline & \multicolumn{2}{|c|}{ TRX(U/L) } & \multicolumn{2}{|c|}{ AFP } & \multicolumn{2}{|c|}{ TRX (U/L) } & \multicolumn{2}{|c|}{ AFP } \\
\hline & $\mathrm{r}$ & $p$ & $\mathrm{r}$ & $p$ & $\mathrm{r}$ & $p$ & $\mathrm{r}$ & $p$ \\
\hline Age/Years & .147 & .318 & .056 & .707 & .062 & 0.37 & -.212 & .149 \\
\hline BMI (Kg//2 & -.190 & .196 & -.146 & .322 & -.044 & .764 & .025 & .863 \\
\hline ALT (IU/L) & .276 & .058 & -.032 & .831 & -.141 & .337 & $.382^{* *}$ & .007 \\
\hline AST (IU/L) & .195 & .184 & -.041 & .783 & -.093 & .528 & $.379^{* *}$ & .008 \\
\hline Albumin (gm/dl) & .067 & .651 & -.114 & .441 & $-.391^{* *}$ & .006 & $.312^{*}$ & .031 \\
\hline T Bilirubin (mg/dl) & .052 & .724 & -.056 & .707 & .204 & .164 & .123 & .403 \\
\hline INR & .264 & .070 & .217 & .139 & $.386^{* *}$ & .007 & .029 & .844 \\
\hline FIB 4 & -.051 & .731 & .142 & .336 & .173 & .240 & .182 & .217 \\
\hline Child score & $.307 *$ & .034 & .248 & .089 & $.651^{* *}$ & $<0.001$ & -.177 & .229 \\
\hline Creatinine (mg/dl) & .131 & .374 & .231 & .115 & .215 & .141 & -.269 & .064 \\
\hline HB (gm/dl) & -.117 & .429 & -.233 & .111 & -.209 & .154 & $.296^{*}$ & .041 \\
\hline TLC $\left(10^{3} / \mathbf{m m}^{3}\right)$ & .034 & .820 & -.090 & .541 & -.063 & .669 & -.069 & .642 \\
\hline Platelet $\left(10^{3} / \mathrm{mm}^{3}\right)$ & .079 & .592 & -.051 & .728 & -.122 & .409 & -.069 & .643 \\
\hline Focal lesion number & $.667^{* *}$ & $<0.001$ & $.548^{* *}$ & $<.001$ & & & & \\
\hline Focal lesion Size & $.636^{* *}$ & $<0.001$ & $.648^{* *}$ & $<0.001$ & & & & \\
\hline
\end{tabular}

ALT, alanine aminotransferase; AST, aspartate aminotransferase; TLC, total leucocytic count.

\section{Discussion}

The progression of liver disease into liver cancer is primarily monitored by serum levels of the oncofetal glycoprotein, alpha-fetoprotein, or the core fucosylated glycoform of AFP (AFP L3), however, its usage has been questioned ${ }^{4}$. Elevated serum thioredoxin levels have been observed in many diseases, including diabetes ${ }^{13}$, chronic kidney disease ${ }^{14}$, severe burn injury ${ }^{15}$ and coronary disease 16. Morever, a variety of malignancies, and evidence suggests that it may be associated with aggressive tumor growth and poor survival ${ }^{8,9,17}$. Our study showed that the level of thioredoxin is significantly higher in patients with HCC in comparison to cirrhotic non-HCC patients. This came following $\mathrm{Li}$ et al., who showed that, the levels of serum thioredoxin in HCC patients were significantly higher than that of healthy subjects, patients with cirrhosis and chronic liver diseases ${ }^{18}$. Also, in agreement with our results, Omran et al., showed that serum levels of thioredoxin were significantly higher in HCC than in patients with liver cirrhosis ${ }^{19}$. In our study, the level of AFP in the HCC group was significantly higher in comparison to the cirrhotic groups. This came in agreement with the result of Yang et al, who revealed that the level of serum AFP in patients with HCC was significantly higher than those of liver cirrhosis and chronic hepatitis patients and healthy controls ${ }^{20}$. This also agreed with El-Edel and his colleagues who showed that serum levels of AFP were significantly elevated in chronic liver diseases and even more elevated in HCC cases with a statistically significant difference ${ }^{21}$. This also came following another study where the AFP serum level showed a highly significant elevation in HCC patients 22. Furthermore, Battaglia et al., also found that the mean plasma concentration of AFP was significantly higher in untreated patients with HCC as compared with patients with chronic liver disease ${ }^{23}$. In this study, thioredoxin at a cutoff value of 198.19 has $85.4 \%$ sensitivity and $89.6 \%$ specificity for differentiating HCC cases from cirrhotic cases with $89.1 \%$ PPV, $86 \%$ NPV, and AUC equal to 0.841 . Moreover, the use of combined AFP and TRX is equal to the use of AFP alone with sensitivity and specificity $(93.8 \%$ and $97.9 \%$ respectively) and AUC equal to 0.99 .

The previous study found that thioredoxin at (cut-off value $120 \mathrm{ng} / \mathrm{ml}$ and AUC 0.79) showed higher sensitivity (74\% vs $29 \%)$ and accuracy (73\% vs 53\%) and lower specificity (71\% vs $100 \%$ ) when compared to AFP at (cutoff value 400U/L and AUC 0.69) for differentiating HCC from cirrhosis ${ }^{19}$. In our study, we found that AFP has a higher sensitivity and specificity than thioredoxin for 
predicting $\mathrm{HCC}$, however, in contrast Li et al, showed that thioredoxin has higher sensitivity and specificity than AFP in the diagnosis of $\mathrm{HCC}$. Li et al, found that the optimal cutoff value of serum thioredoxin levels as an indicator for auxiliary diagnosis of HCC was projected to be $20.5 \mathrm{ng} / \mathrm{mL}$, which yielded a sensitivity of $84.3 \%$ and a specificity of $91.8 \%$, with the area under the curve at 0.946 (95\% CI, 0.923-0.969); at optimum cutoff value for AFP $18.5 \mathrm{ng} / \mathrm{mL}$ (AUC $0.878,95 \%$ CI: $0.841-0.914$ ) the sensitivity was $78.4 \%$, the specificity of $81.3 \%$ ). The authors also suggested an advantage of thioredoxin over AFP as a serum marker for the detection of $\mathrm{HCC}$, especially for patients with very early HCC and AFP-negative status ${ }^{18}$. This discrepancy in results regarding thioredoxin may be explained by the diversity in population and ethnicity, genetic variations, use of different cut-off values, or variable etiology of $\mathrm{HCC}^{24}$.

In this study, in HCC cases, thioredoxin has a statistically significant positive correlation with Child-Pugh classification, focal lesion number, and size. AFP have also statistically significant positive correlation with focal lesion number and size. While in the cirrhosis group, thioredoxin has a statistically significant positive correlation with ChildPugh classification, INR, and negative correlation with albumin. AFP have also statistically significant positive correlation with ALT, AST, albumin, and hemoglobin. Similar results were reported by $\mathrm{Li}$ et al, who showed that there was a significant correlation between thioredoxin concentrations and tumor size, Child-Pugh class, or tumor stage. The level of thioredoxin tended to increase as the liver disease progressed from Child-Pugh class A to ChildPugh class $\mathrm{C}$ as well as tumor stage from I to IV. For comparison, AFP values of different tumor sizes or differentiation status, or liver function were also analyzed. There was no significant correlation were obtained ${ }^{18}$. Our study has some limitations including, a single-center study and the small number of patients.

\section{Conclusion}

Thioredoxin (TRX) is a novel biomarker that revealed good sensitivity in the early prediction of the development of HCC on top of liver cirrhosis.

\section{References}

1. Elbaz, S., Mousa, N., Besheer, T., et al. (2020). Malondialdehyde and C-reactive protein as prognostic markers of hepatocellular carcinoma. British journal of biomedical science, 77(2), 94-96.

2. Elgamal, S., Ghafar, A. A., Ghoneem, E., et al. (2018). Characterization of patients with hepatocellular carcinoma on the way for early detection: one center experience. The Egyptian Journal of Internal Medicine, 30(4), 231-238.

3. Plentz, R. R., and Malek, N. P. (2015). Early detection of hepatocellular carcinoma: how to screen and follow up patients with liver cirrhosis according to the GERMAN S3 guideline? Diagnostics, 5(4), 497-503.

4. Mohammed Amin Mohammed., Hany Shabana., Tarek Sheta., et al. (2017). Vitamin D Receptor Gene Polymorphisms as a Predictive Risk Factor for Hepatocellular Carcinoma Development and Severity in Chronic Hepatitis B. International Journal of Cancer Research, (13): 26-35.

5. Trevisani, F., D'Intino, P. E., Morselli-Labate, A. M., et al. (2001). Serum $\alpha$-fetoprotein for diagnosis of hepatocellular carcinoma in patients with chronic liver disease: influence of $\mathrm{HBsAg}$ and anti-HCV status. Journal of hepatology, 34(4), 570-575.

6. Mousa, N., Gad, Y., Abdel-Aziz, A., et al. (2012). Increased-Fetoprotein Predicts Steatosis among Patients with Chronic Hepatitis C Genotype 4. International journal of hepatology, 2012.

7. Abdel-Razik, A., Mousa, N., Abdel-Aziz, M., et al. (2016). Elevated serum $\alpha$-fetoprotein levels in patients with chronic hepatitis C virus genotype 4: not the end of the story. European journal of gastroenterology \& hepatology, 28(3), 313-322.

8. Kondo, N., Nakamura, H., Masutani, H., et al. (2006). Redox regulation of human thioredoxin network. Antioxidants \& redox signaling, 8(9-10), 1881-1890.

9. Welsh, S. J., Bellamy, W. T., Briehl, M. M., et al. (2002). The redox protein thioredoxin-1 (TRX-1) increases hypoxia-inducible factor $1 \alpha$ protein expression: TRX-1 overexpression results in increased vascular endothelial growth factor production and enhanced tumor angiogenesis. Cancer research, 62(17), 5089-5095.

10. Miyazaki, K., Noda, N., Okada, S., et al. (1998). Elevated serum level of Thioredoxin in patients with Hepatocellular Carcinoma. Biotherapy, 11(4), $277-$ 288.

11. Bruix, J., and Sherman, M. (2005). AASLD practice guideline. Management of hepatocellular carcinoma. Hepatology, 42(5), 1208-1236.

12. Papadopoulos, N., Vasileiadi, S., Papavdi, M., et al. (2019). Liver fibrosis staging with combination of APRI and FIB-4 scoring systems in chronic hepatitis $\mathrm{C}$ as an alternative to transient elastography. Annals of gastroenterology, 32(5), 498.

13. Kakisaka, Y., Nakashima, T., Sumida, Y., et al. (2002). Elevation of serum thioredoxin levels in patients with type 2 diabetes. Hormone and metabolic research, 34(03), 160-164.

14. Tsuchikura, S., Shoji, T., Shimomura, N., et al. (2010). Serum C-reactive protein and thioredoxin levels in subjects with mildly reduced glomerular filtration rate. BMC nephrology, 11(1), 1-8.

15. Abdiu, A., Nakamura, H., Sahaf, B., et al. (2000). Thioredoxin blood level increases after severe burn injury. Antioxidants \& redox signaling, 2(4), 707716. 
16. Miyamoto, S., Kawano, H., Sakamoto, T., et al. (2004). Increased plasma levels of thioredoxin in patients with coronary spastic angina. Antioxidants and Redox Signaling, 6(1), 75-80.

17. Kakolyris, S., Giatromanolaki, A., Koukourakis, M., et al. (2001). Thioredoxin expression is associated with lymph node status and prognosis in early operable non-small cell lung cancer. Clinical cancer research, 7(10), 3087-3091.

18. Li, J., Cheng, Z. J., Liu, Y., et al. (2015). Serum thioredoxin is a diagnostic marker for hepatocellular carcinoma. Oncotarget, 6(11), 9551.

19. Omran, M. M., Farid, K., Omar, M. A., et al. (2020). A combination of $\alpha$-fetoprotein, midkine, thioredoxin and a metabolite for predicting hepatocellular carcinoma. Annals of hepatology, 19(2), 179-185.

20. Yang, L., Xu, Q., Xie, H., et al. (2016). Expression of serum miR-218 in hepatocellular carcinoma and its prognostic significance. Clinical and Translational Oncology, 18(8), 841-847.

21. EI-Edel, R. H., Tawfik, G. A., Noreldin, R. I., et al. (2018). Evaluation of serum Midkine as a novel marker in hepatocellular carcinomas. Menoufia Medical Journal, 31(3), 1094.

22. Baghdady, I., Fouad, F., Sayed, M., et al. (2014). Serum markers for the early detection of hepatocellular carcinoma in patients with chronic viral hepatitis C infection. Menoufia Medical Journal, 27(3), 544.

23. Battaglia, S., Benzoubir, N., Nobilet, S., et al. (2009). Liver cancer-derived hepatitis $\mathrm{C}$ virus core proteins shift TGF-beta responses from tumor suppression to epithelial-mesenchymal transition. PloS one, 4(2), e4355.

24. Omran, M. M., Emran, T. M., Farid, K., et al. (2016). An easy and useful noninvasive score based on $\alpha-1$ acid glycoprotein and c-reactive protein for diagnosis of patients with hepatocellular carcinoma associated with hepatitis c virus infection. Journal of Immunoassay and Immunochemistry, 37(3), 273288. 